\title{
Delayed Detonation at a Single Point in Exploding White Dwarfs
}

\author{
E. Livne
}

Racah Institute of Physics, The Hebrew University, Jerusalem 91904, Israel

Received — 


\begin{abstract}
Delayed detonation in an exploding white dwarf, which propagates from an off-center transition point, rather than from a spherical transition shell, is described and simulated. The differences between the results of $2 \mathrm{D}$ simulations and the 1D case are presented and discussed. The two dimensional effects become significant in transition density below $3 \times \rho_{7}$, where the energetics, the production of Fe group elements and the symmetry of the explosion are all affected. In the $2 \mathrm{D}$ case the explosion is less energetic and less $\mathrm{Ni}$ is produced in the detonation phase of the explosion. For low transition density the reduction in Ni mass can reach 20-30 percent. The asymmetry in abundances between regions close to the transition point and regions far from that point is large, and could be a source to polarization patterns in the emitted light. We conclude that the spatial and temporal distribution of transition locations, is an important parameter which must be included in delayed detonation models for Type Ia supernovae.
\end{abstract}

Subject headings: hydrodynamics-nucleosynthesis,abundancessupernovae:general 


\section{Introduction}

The delayed detonation (DD) model is a successful model for type Ia supernovae (SnIa), as it meets most of the constraints imposed by observations (Khokhlov 1991a, Khokhlov 1991b). The DD model assumes a transition from a deflagration to a detonation (DDT) at some critical density (of the fuel ahead of the front), after a significant expansion of the WD during a slow deflagration phase. This critical density is limited to a fairly

small range around $2 \times 10^{7} \mathrm{~g} \mathrm{~cm}^{-3}$ by observational constraints (HöHlich 1995, Höflich et al. 1995, Höflich \& Khokhlov 1996). It is also the density at which turbulence can destroy the flame (Khokhlov et al. 1997). In this paper I do not discuss the difficult problem of the DDT itself, but rather examine the geometrical effects of asymmetrical transition. The vast majority of numerical simulations that have been performed up to now assumed spherical symmetry, where the transition to detonation occurs in a spherical shell. Only a few simulations have tried to include multi dimensional effects Arnett \& Livne 1994a, Arnett \& Livne 1994b), but in those works the geometrical effects of the location of the transition, on the explosion, were not considered. One can judge different model by comparing the masses and velocities of different products to observations. In particular, the chemical composition of the ejecta versus velocity is an important imprint of any model. A typical common problem to spherical DD simulations is a small, but not insignificant, amount of slow moving intermediate mass elements $(3000-4000 \mathrm{~km} / \mathrm{sec})$, around the transition point (Höflich 1995, Lwamoto et al. 1999). Such slow moving intermediate mass elements are hard to observed, and they do not show in theoretical spectra.

The purpose of this letter is to go beyond the 1D case by studying the more probable scenario, in which the transition to detonation occurs off-center in an isolated point, located near on the deflagration surface. Then the hydrodynamical problem is not spherically symmetric, but rather axially symmetric around the line which passes through the center 
and the transition points. In exploring this scenario we are motivated by two facts - first, when the density ahead of the front drops to the critical density the radius of the (spherical) front is roughly a third of the total radius, and second, DDT occurs on scales much smaller than the radius of the star, or even the radius of the front (Khokhlov et al. 1997). Hence, transition at a point is a good approximation to any realistic scenario. Moreover, there are still severe doubts as to the robustness of DDT in thermonuclear flames (Niemeyer 1999), so DDT in a spherical shell of such a large radius seems very improbable. The case we are studying here, off-center point transition, is the most extreme asymmetric case, while transition in a spherical shell is the most symmetric case. Assuming one point transition, The detonation front propagates from the transition point inside the unburnt shell and around the already burnt core, in a manner similar to the detonation in a helium shell (Livne \& Glasner). Although the time needed for this detonation wave to consume the whole star is much shorter than any other dynamical time (roughly $0.5 \mathrm{sec}$ ), we can not rule out the possibility of DDT in several unsynchronized points. This however would require 3D simulations which are currently beyond our computing capabilities. Regarding the symmetry of the explosion, transition in several points would behave as an intermediate

case between the spherical case and the single off-center case. I shall refer to this possibility in the concluding remarks at the end of the paper. In the next two section we describe briefly the hydrodynamics, the simulations and mainly the observational results of this unexplored scenario.

\section{The models and the numerical techniques}

The simulations are performed using the $1 \mathrm{D}$ and $2 \mathrm{D}$ versions of the hydro code VULCAN (Livne 1993), supported by an accurate and tabulated equation of state and a reaction network that is based upon the alpha network (Thielemann, private 
communication). The initial model consists of a cold, almost isentropic WD of $1.39 M_{\odot}$, central density of $2 \times \rho_{9}$ and a constant $\mathrm{C}-\mathrm{O}$ ratio of 1 . The deflagration phase is performed in a 1D spherically symmetric simulation, using a front speed of 2 percent of the local sound speed (Khokhlov 1991a). In coarse grids there are nearly 200 uneven radial zones, where the resolution around the transition radius is $\delta m=0.01 \times M_{\odot}$, which corresponds to a zone size of $20 \mathrm{~km}$. We consider 3 cases of transition densities - $\rho_{t r}=2 \rho_{7}$ (case A), $\rho_{t r}=3.5 \rho_{7}$ (case B) and $\rho_{t r}=5 \rho_{7}$ (case C), for which the deflagration front radius is $r_{f}=1880 \mathrm{~km}$, $1500 \mathrm{~km}$ and $1300 \mathrm{~km}$ respectively. For each case, the deflagration stage terminates when the density ahead of the front reaches the critical density.

In order to be able to compare the two cases I deliberately choose to start the detonation phase with a spherical undisturbed configuration, that is produced by the 1D code. By simulating the deflagration phase in 1D we neglect the complicated structure of the front due to Rayleigh-Taylor instability. Hence, the results presented in this work must not be considered as a new realistic DD model. They measure only the effects of the geometry of the transition on the explosion products. This simplifications will be removed in future work, where the deflagration phase will be done in 3D. The location of the transition point, however, will remain a free parameter, as the physics of the transition is not well understood, and in any case DDT occurs on scales much smaller than the resolution of any realistic simulation.

At the end of the deflagration stage the density behind the front is almost half $\rho_{t r}$. In the lower density cases, intermediate mass elements ( $\mathrm{Si}$ ) burn very slowly and the typical Si-scale of the front exceeds $100 \mathrm{~km}$ (Gamezo et al. 1999). In 1D simulations the transition to detonation at the front pushes the unburnt matter ahead of the front forwards, and emits a rarefaction wave into the partially burnt matter behind it. As a result, burning of intermediate mass elements near the transition radius is never completed. In our 1D 
simulation the large scales (Si scales) of the front $(\approx 100 \mathrm{~km})$ around the transition radius

are not finely resolved, but the results with finer zones are not very different. The transition to detonation is done numerically by increasing the front speed around the transition point (the intersection of the front with the right axis in fig 1a) to the sound speed. I simulate the detonation phase both in $1 \mathrm{D}$, in $2 \mathrm{D}$ with shell ignition (a validity check) and in $2 \mathrm{D}$ with DDT at a single point. In the 2D grid it takes about 20-30 zones for the sliding front to reach a quasi stable Chapman-Jouguet (CJ) state. The corresponding distance from transition to a CJ state is much larger in the simulation than in realty, where it is known from experiments that this distance is roughly 10,000 times the width of the front. The $2 \mathrm{D}$ simulations are terminated 1 second after DDT, where all burning processed are already completed. Relatively small changes in abundances distribution are possible after this time.

\section{Results from $2 \mathrm{D}$ simulations}

The main structure of the detonation front in case A is seen in figure 1a. The most obvious feature, which is absent in 1D simulations, is the inward moving oblique shock, induced by the sliding detonation. This shock raises the density of the partially burnt fuel, below the front radius, by almost a factor of 2 . However, despite a significant enhancement of the burning rates, intermediate mass elements are not exhausted. When the detonation front converges to the axis of symmetry on the far side it becomes stronger, and a Mach reflection is formed after it meets the axis. This causes the ejection of a jet of burnt matter, mostly Ni, along the axis. In all three cases, less iron group, and more silicon group species, are produced in the $2 \mathrm{D}$ simulation, compared to the $1 \mathrm{D}$ case. The Ni productions in the $1 \mathrm{D}$ simulations for cases $\mathrm{A}, \mathrm{B}, \mathrm{C}$ are $0.68 M_{\odot}, 0.97 M_{\odot}$ and $1.07 M_{\odot}$, while in the $2 \mathrm{D}$ simulations the corresponding numbers are $0.44 M_{\odot}, 0.85 M_{\odot}$ and $0.99 M_{\odot}$. Si productions in the 1D cases are $0.27 M_{\odot}, 0.16 M_{\odot}$ and $0.12 M_{\odot}$ while in the $2 \mathrm{D}$ cases they are $0.36 M_{\odot}, 0.19 M_{\odot}$ and 
$0.14 M_{\odot}$. We see that the two dimensional effect becomes stronger with decreasing transition density.

\section{EDITOR: PLACE FIGURE 1 HERE.}

There are two interesting hydrodynamical effects which work together towards a less energetic explosion and less efficient combustion. The first one is related to the direction of the detonation front with respect to the density gradient. In the spherical case, the detonation is not a CJ detonation but a strong detonation. This is a result of the decreasing density gradient which accelerates the shock beyond its CJ values. In the $2 \mathrm{D}$ case, the angle between the detonation front and the density gradient varies from point to point. Close to the nearly stalled deflagration front the detonation slides along the burnt core in a constant density background, so it travels with almost CJ states. Even far from the transition shell the detonation is not oriented in the radial direction (see figure 1a), so it is weaker than the spherical detonation front.

The second hydrodynamical effect is the continuous expansion of the star, during the travel of the detonation front around the transition front. It turns out that the expansion velocity near the front (nearly $800-1000 \mathrm{~km} / \mathrm{sec}$ ) is enough to cause an increase of the front radius by $20-25$ percent during the travel of the detonation around the burnt core. Since in a subsonic expansion the density changes like $r^{-3}$, the fuel density head of the detonation front drops by almost a factor of two during this time, and consequently the burning temperature is also lower. As a whole, the fuel density ahead of the detonation drops continuously as it travels around, and by the time it reaches the far side the fuel density is roughly half its original value. In addition, this effect causes a pronounced asymmetry in the abundances of Fe group elements between the near and far sides along the axis. The effect is clearly seen in figure $1 \mathrm{~b}$, in which we present the average atomic number at the end 
of the $2 \mathrm{D}$ simulation of case $\mathrm{A}$.

\section{Conclusions and Discussion}

The scenario of deflagration to detonation transition (DDT) in a single off-center point, rather than in a shell, have been considered. In order to be able to compare the two cases I deliberately choose to neglect the Rayleigh-Taylor instability during the deflagration phase, and to start the detonation phase with a spherical undisturbed configuration, that was produced by the $1 \mathrm{D}$ code. Although this way imposes unrealistic initial conditions for the detonation phase, it enables one to isolate the net effect of the geometry of the transition. Hence, the results presented in this work must not be considered as a new realistic DD model. They measure only how the geometry of the transition affects the explosion products. The results show significant differences between the 1D case and the 2D case, in the amounts and in the distributions of the explosion products. This implies that the spatial and temporal distribution of DDT points is an important parameter for modeling SnIa's. In the case of DDT in an off-center point, located on the deflagration front, less $\mathrm{Ni}$ is produces compared to the spherical case, as a result of a weaker detonation front and the continuously decreasing density background. The difference increases as the transition density becomes lower, and can reach a reduction of 20-30 percent in the Ni production at $\rho_{t r}=2 \rho_{7}$. In addition, the $2 \mathrm{D}$ effects tend to widen the range of intermediate mass elements in velocity space, and would lower the minimal velocity for such elements. This is an important consideration for a consistent comparison between models and observations (Lwamoto et al. 1999). For low transition density $\left(2 \times 10^{7} \mathrm{~g} \mathrm{~cm}^{-3}\right)$ the intermediate mass elements produced at the end of the deflagration phase do not disappear in the $2 \mathrm{D}$ simulation. The oblique shock induced by the sliding detonation upon the burnt core is just too weak. This conclusion however needs verification with finer zones than used here, near 
the edge of the deflagration front.

Another important outcome of the scenario is a pronounced asymmetry in the $\mathrm{Ni}$ abundance along the axis, and again, this asymmetry increases with decreasing transition density. Recent analysis of polarization in some SnIa's provides evidences for asymmetry in the explosion products (Wang et al. 1997). Modeling those polarization patterns suggest that the asphericity is attributed to a region between a slow moving, almost spherical, Ni core and a spherical Si layer. This is exactly the feature seen in fig $1 \mathrm{~b}$, where the products of the deflagration phase form a spherical Ni core, and the products of the detonation phase form aspherical region of partial burning, above which lies an almost spherical Si shell. For transition density above $3.5 \times 10^{7} \mathrm{~g} \mathrm{~cm}^{-3}$ the asphericity almost disappear except for the narrow jet on the far axis. Consequently, the scenario of DDT in a single point can be founded if a relation between $\mathrm{Ni}$ mass and asphericity (polarization) will be found in observations. Theoretically, a more systematic study of the range $2 \rho_{7}<\rho_{t r}<3 \rho_{7}$ is required.

It is clear that in the scenario presented here, the transition density that would give a best fit to observations is higher than in the 1D case. This however will be different in the pulsational delayed detonation model (PDD), if DDT occurs during the contraction phase of the pulse. Thus, we expect that the differences between the direct DD and the PDD models will be larger in the scenario of point transition than in the case of a spherical transition. Several other complications may alter somewhat the results. First, in reality and in more realistic multi dimensional simulations of the deflagration phase, the deflagration front will be distorted and eventually develop disconnected burning pockets (Niemeyer and Woosley 1997). This will cause the detonation front to move in an inhomogeneous medium and complicated features of shock interactions are possible, mainly close to the edge of the deflagration front. The main uncertainty still is the location of the transition point, and 
even in future 3D simulations this location (s) will remain a free parameter that strongly affects the explosion products.

Secondly, several points may run to detonation before the first detonation wave has completed its propagation around the star. This effect can alter somewhat the lowest modes in the angular expansion of the asymmetry. It will require synchronization of the transition at different points to a time interval of roughly 0.5 second. The interaction between detonation waves that propagate from different transition points would form regions of enhanced burning (Mach branches), and the effect is expected to produce much larger inhomogeneity than the instability described in Gamezo et al. 1999. The effect can disappear only if the distance between different and simultaneous transition points, is less than $\mathrm{E}_{\text {crit }}$ - the characteristic distance needed to form a stable CJ detonation. In our case, $L_{\text {crit }}$ is of the order of several meters up to a few kilometers (Khokhlov et al. 1997), so one needs thousands of simultaneous transitions in order to suppress shock interactions. This seems very improbable. Finally, both complications mentioned above, and many other factors of the DDT mechanism, act in a chaotic manner and will give rise to significant variations between SnIa events.

This work was motivated by P.Höflich during my visit to Aspen Center of Physics in summer 1999. I also thank P.Höflich for enlightening comments and suggestions to the first draft. 


\section{REFERENCES}

Arnett,W.D. \& Livne,E.,1994,ApJ,427,314

Arnett,W.D. \& Livne,E.,1994,ApJ,427,314

Gamezo,V.N.,Wheeler,J.C.,Khokhlov,A.M. and Oran,E.S., 1999,ApJ,512,827

Khokhlov, A.M., 1991a, A\&A 245, 114

Khokhlov, A.M., 1991b, A\&A 245, L25

Khokhlov, A.M.,1995,ApJ,449,695

Khokhlov,A.M.,Oran,E.S. and Wheeler,J.C.,1997,ApJ,478,678

Höflich,P.,1995,ApJ,443,89

Höflich,P., Khokhlov,A.M. and Wheeler,J.C.,1995,ApJ,444,831

Höflich,P. and Khokhlov,A.M,1995,ApJ,457,500

Livne,E., \& Glasner,A.S.,1990, ApJ,361,244

Livne,E.,1993,ApJ,412,634

Niemeyer,J.C., 1999, astro-ph/9906142

Niemeyer,J.C. and Woosley,S.E.,1997, ApJ,475,740

Iwamoto,K.,Brachwitz.,F.,Nomoto,K.,Kishimoto,N.,Hix,W.R. and Thielemann,F.K., 1999, ApJ, in press

Wang,L.,Wheeler,J.C. and Höflich,P.,1997,ApJ,476,L27 
Fig. 1.- a-(up) Pressure during the detonation phase, b-(down) Average atomic number at $\mathrm{t}=1$ sec after DDT 
This figure "fig1.gif" is available in "gif" format from: http://arxiv.org/ps/astro-ph/9910471v1 